Results A total of 126 patients tested positive for HIV in KAMC-Riyadh during the study period. 53 of these patients were screened for hepatitis $\mathrm{B}$, of whom four were positive; 55 were screened for hepatitis C, of whom four were also positive, and 72 patients were screened for tuberculosis, of whom seven were positive. The remainder of the patients were never screened for any of the diseases.

Conclusion More than $40 \%$ of patients with HIV were not screened for either hepatitis B, hepatitis C, or tuberculosis, which are the most common co-infections with HIV. These diseases may progress quickly and could be managed if discovered early or prevented. These results demonstrate the need for a comprehensive HIV program that involves early screening and protection against all infectious diseases.

\section{PREVENTION OF MOTHER-TO-CHILD TRANSMISSION OF HEPATITIS B IN KING ABDULAZIZ MEDICAL CITY, RIYADH}

Sara AlMunif, Nouf Al Enizi, Majid AlShamrani. Department of Infection Prevention and Control, King Abdulaziz Medical City

\subsection{6/bmjoq-2019-PSF.72}

Background Hepatitis B continues to affect many people in Saudi Arabia, causing a huge burden on the healthcare system for treating patients and preventing spread of the disease. Because hepatitis B is not curable, transmission of infection can only be prevented by vaccinating those susceptible, paying special attention to children of positive mothers. Guidelines suggest that all infants born to positive mothers should receive both the hepatitis $\mathrm{B}$ vaccine and immune globulin within 12 hours of birth. In addition to the remaining two doses of the hepatitis vaccine, infants should also be tested for hepatitis B 3 to 6 months following the last dose of vaccine. The aim of this study was to identify if international guidelines were followed after delivery in hepatitis B-positive mothers at KAMCRiyadh.

Methods This was a retrospective analysis of pregnant mother's positive to hepatitis B who gave birth in KAMC-Riyadh during 2016-2017. Data regarding administration of vaccine and immune globulin, completion of vaccine series, and follow-up testing were extracted from the child's medical record. Results During the 2-year study period, a total of 105 hepatitis B-positive mothers gave birth, and all infants were administered the birth dose of the hepatitis B vaccine; however, only 55 children were documented to have completed the threedose series of the vaccine. Only 101 infants were administered immune globulin after birth, and only two children were tested for the virus.

Conclusion Infants born to hepatitis B-positive mothers do not receive special attention or follow-up. It is not known why four infants did not receive immune globulin; however, unclear documentation of the virus in the mother's file during pregnancy or delivery may be a reason. Many of these children did not receive their full vaccine series and almost all were not tested for the virus. These numbers demonstrate the need for a strategic plan for the management and follow-up of infants born to hepatitis B-positive mothers.

\section{3 JOURNEY TO CBAHI ACCREDITATION AT NATIONAL GUARD HEALTH AFFAIRS}

Hadeel Alderaihem, Abdullah Yaqub, Jade Laist, Julita Camorista, Kokila Periasamy, Romel Casilla. Quality and Patient Safety Riyadh, King Abdulaziz Medical City Riyadh

\subsection{6/bmjoq-2019-PSF.73}

Background MNGHA facilities have been accredited by the Joint Commission International (JCI) since 2006. Leaders in MNGHA believe that the accreditation process is a tool to improve the quality and patient safety in the hospital, applying evidence-based standards in performance with focus on the outcomes. A decision was made by the leadership to attain a national accreditation certificate (Central Board for Accreditation of Healthcare Institutions, CBAHI). In 2016, MNGHA facilities had the first experience with CBAHI through the ESR program. All facilities were visited by two surveyors to evaluate the compliance of MNGHA hospitals with 20 Essential Safety Requirements, as this was a national project to improve patient safety in all healthcare facilities in the Kingdom of Saudi Arabia. After this experience, MNGHA leaders were interested to submit all MNGHA facilities for a national accreditation represented by CBAHI in addition to international accreditation represented by JCI. All MNGHA facilities were scheduled for CBAHI accreditation in 2018, the Central Region in April 2018, Western Region in May, Al Ahsa in July, Dammam in August, and Madina in October, which was postponed to February 2019.

Methods A team consisting of 18 certified CBAHI surveyors was scheduled to conduct the mock survey for all regions at least 3 months prior to the real survey. MNGHA leadership fully supported the project by providing the required resources to achieve the desired outcome from the project.

Results All MNGHA facilities had their mock survey by the MNGHA's surveyors with excellent feedback with the following outcomes: tremendous learning opportunities were created during the mock survey, improve the intraregional interaction by sharing experiences, create a resource team in the system from different specialties for CBAHI accreditation. Common findings were identified among all MNGHA Regions, which led to some changes at the corporate level of MNGHA. Moreover, the impact of the project on the real survey of all hospitals was remarkable; the MNGHA's hospital scores were considered as extraordinary results:

Facility Score

KAMC - Central Region 96.40\%

KAMC - Western Region 92.27\%

KAH- Al Ahsa 94.66\%

IABFH Dammam 96.75\%.

Conclusion Building capacity is crucial in improving the system and process, utilizing local resources and appreciating staff skills and knowledge is the way to achieve the culture of a learning organization, connecting people from different systems and settings will create a culture of learning, sharing successful experiences of overcoming challenges standard with the same resources will highly impact a positive outcome in the real survey. 